\title{
Celiac Disease Treatment: Is It the Chicken or the Egg Yolk?
}

\author{
Valentina Discepolo ${ }^{1,2} \cdot$ Stefano Guandalini ${ }^{3}$
}

Published online: 13 February 2017

(c) Springer Science+Business Media New York 2017

\section{Background and Significance}

Celiac disease (CD), one of the most common food-related lifelong diseases, is prevalent worldwide. Its incidence, like that of many other autoimmune disorders, is apparently increasing over time, although recently, it may possibly be plateauing, at least in the USA [1]. From a pathogenic standpoint, $\mathrm{CD}$ is the result of a complex inflammatory immune response arising in the small intestinal mucosa of genetically susceptible individuals upon the ingestion of dietary gluten.

At present, a strict lifelong gluten-free diet (GFD) is the only valid and effective treatment for CD [2]. Good compliance with a GFD is usually associated with the normalization of the intestinal mucosal lesions and alleviation of symptoms and, when nutritionally balanced, does not lead to adverse effects. Nevertheless, it remains unclear how much the GFD helps preventing the development of other CD-associated disorders such as autoimmune thyroiditis and type 1 diabetes [4].

Despite its efficacy, adherence to GFD remains a difficult goal for many patients due to its cost and social restrictions, especially for adolescents and young adults. Often, commercially available gluten-free products are less palatable and scarcely available as compared to their gluten-containing counterparts. Of note, patients who do not

Stefano Guandalini

sguandal@uchicago.edu

1 Department of Translational Medical Sciences, Section of Pediatrics, University of Naples Federico II, Naples, Italy

2 CeInGe, Biotecnologie Avanzate, Naples, Italy

3 Department of Pediatrics, University of Chicago Celiac Disease Center, University of Chicago, Chicago, IL, USA strictly follow the diet may encounter many complications and may be exposed to a persistent autoimmune response whose effects could be potentially dangerous [3]. Moreover, a small, but not irrelevant, proportion of adult patients with $\mathrm{CD}$ fail to respond to the GFD, developing a complication known as refractory $\mathrm{CD}$ requiring specific immunotherapy [4]. The need for alternative therapies to GFD arises from these observations.

A better understanding of $\mathrm{CD}$ pathogenesis has led in the past years not only to improvements in the diagnosis of $\mathrm{CD}$, but also to the development of new therapeutic approaches ranging from strategies to reduce intestinal permeability, to supplement or replace GFD enzyme supplementation therapy, to blockers of gliadin deamidation or gluten peptide presentation to immune cells [5].

Based on their mechanisms of action and the rationale behind their conception, the therapeutic approaches could be divided into:

- alternative cereals

- gluten-detoxifying strategies

- inhibitors of gliadin transport across the intestinal barrier

- immune modulators

Alternative dietary strategies include non-toxic, genetically modified cultivars as well as gluten digestion during food processing. Among gluten-detoxifying strategies, gluten digestion using probiotics, oral proteases, and gluten-sequestering polymers that prevent the interaction of undigested gluten peptides with the intestinal mucosa could be used together with a gluten-containing diet or as a supplement to the GFD in order to avoid damage consequent to inadvertent gluten intake.

Preventing the transport of gliadin peptides from the lumen to the small intestinal lamina propria could be 
achieved using modulators of tight junctions (such as larazotide [6]) or blocking transcellular gliadin transport. Finally, several immune modulatory strategies have been advanced for CD treatment, from anti-interleukin (IL)-15 antibodies for refractory $\mathrm{CD}$ patients to drugs blocking deamidation or presentation of gliadin peptides to $\mathrm{T}$ cells. Importantly, efforts have been made to develop a definitive cure for CD by a peptide-based vaccination strategy [7] or suppression of the inflammatory immune response with hookworm infections [8]. The restoration of tolerance toward orally ingested gluten is currently the only approach that might lead to a cure, rather than treatment, of the disease. Some of these novel therapeutic options have already entered clinical trials, but before marketing, more testing in large cohorts of patients is required.

Most of the issues related to the concept and development of novel therapeutic strategies depend on the observations that $C D$ is a benign disorder and that GFD has a highly favorable safety and tolerability profile; thus, any other approach will need to have an acceptable benefit/risk ratio. Evaluation of efficacy, though problematic, requires a full evaluation including serology, histological assessment of the small intestinal mucosa, careful evaluation of clinical symptoms, if present, and assessment of quality of life. In this respect, obstacles to progress include the lack of an animal disease model for preclinical testing of noninvasive surrogate markers for gluten-induced damage to the small bowel, and the lack of effective CD-related symptoms outcome measures. An unambiguous evaluation of efficacy is an even more difficult task when testing supplements to the GFD, given that the diet itself ameliorates all of the above-mentioned measures.

At present, it seems that therapeutic options aiming at detoxifying dietary gluten may represent the best option for patients exposed to cross-contamination and inadvertent gluten intake.

\section{Discussion}

In a very preliminary pilot study published in this issue of Digestive Diseases and Sciences, Sample and colleagues report their experience with AGY, a specific chicken egg yolk-derived immunoglobulin (IgY) against wheat gliadin on a cohort of ten patients with biopsy-proven $\mathrm{CD}$ with the aim of assessing its safety in adult CD individuals already consuming a GFD for at least 6 months [9]. Previous preclinical studies performed by the same authors demonstrated that this polyclonal antibody is able to neutralize gliadin in the presence of simulated gastrointestinal fluids in mice.

Despite several limitations, principally the very small number of patients enrolled and the lack of blinding and of a placebo arm, the study does provide preliminary evidence of a good safety profile and tolerability of the compound. All ten patients enrolled had good compliance, consuming the drug at every meal and, according to the authors, displaying a pattern of improvement of clinical symptoms as well as an improvement trend (though within the normal range) in the levels of $\mathrm{CD}$-specific anti-tissue transglutaminase antibodies.

Considering in aggregate the data collected in preclinical studies along with data provided by this pilot study, AGY could represent a promising future therapeutic strategy in patients on a GFD in order to avoid cross-contamination or inadvertent gluten exposure in specific social contexts such as traveling abroad or eating in places where the gluten content of the food is uncertain. Thus, AGY may represent a useful tool for $\mathrm{CD}$ patients consuming a strict GFD who require further help in order to reduce the risk of inadvertent gluten exposure.

Since the evidence provided so far must be considered very preliminary, the need for more studies of AGY appears evident. Indeed, this single-arm, open-label, pilot study was performed on a too small and not gender-balanced cohort including only ten adult subjects, nine of whom were females. A more comprehensive study design should include a better balance of genders and be powered enough with a larger number of subjects in order to show conclusive evidence of efficacy. Clearly, a placebo arm is further required to assess whether the clinical improvement described by the authors on this small group of subjects could be attributed merely to a placebo effect rather than to the drug itself. Adding a detailed description of the daily gluten intake and the evaluation of intestinal inflammatory response would be needed to document the effectiveness of this preparation in maintaining mucosal integrity, an outcome measure indispensable for a drug with such claims.

Given those limitations, acknowledged even by the authors, it is difficult to predict the impact AGY will have in the future in which numerous alternative therapies for $\mathrm{CD}$ patients will be available. Looking at the future, it is encouraging that the authors, supported by these preliminary findings, are planning to design a wider prospective study that should address most of the issues raised here. To this objective, we suggest that clear outcomes be identified; in particular, the amount of gluten that could be neutralized by a defined amount of the compound should be carefully assessed so as to enable a reasonable definition of the possible uses of the drug in practice.

Once the safety and efficacy of AGY are confirmed in a larger cohort, it would then be interesting to investigate whether this preparation could be used for other glutenrelated disorders in light of the remarkable increase in the use of a gluten-free diet outside of CD [1], in particular for subjects affected by the still vague entity termed non-celiac 
gluten sensitivity (NCGS) or wheat intolerance syndrome (WIS) [10], characterized by a wide range of symptoms elicited by gluten that may be "dose-related." We could in fact hypothesize that neutralizing even a fraction of the ingested gliadin could be beneficial in terms of reducing clinical symptoms in the subset of these subjects that truly react to gluten.

\section{Summary and Future Directions}

The work performed by Sample and colleagues comes on the heels of a number of research attempts to find therapeutic alternatives to GFD or at least to improve the quality of life of CD patients already following a GFD by providing gluten-neutralizing strategies. AGY could enter the latter group of therapies, thus potentially representing an adjuvant to the GFD. Nevertheless, further more robust studies are required to confirm its safety, test its efficacy, in particular with regard to the maintenance of the intestinal mucosal integrity, and define the details of its administration to $\mathrm{CD}$ subjects. The scientific community focusing on advances in $\mathrm{CD}$ will certainly look with great anticipation to a wider, doubleblinded trial including a placebo arm and specific defined outcomes.

\section{References}

1. Choung RS, Unalp-Arida A, Ruhl CE, Brantner TL, Everhart JE, Murray JA. Less hidden celiac disease but increased gluten avoidance without a diagnosis in the United States: findings from the national health and nutrition examination surveys from 2009 to 2014. Mayo Clin Proc. 2017;92:30-38.

2. Norstrom F, Sandstrom O, Lindholm L, Ivarsson A. A gluten-free diet effectively reduces symptoms and health care consumption in a Swedish celiac disease population. BMC Gastroenterol. 2012;12:125.

3. Shannahan S, Leffler DA. Diagnosis and updates in celiac disease. Gastrointest Endosc Clin N Am. 2017;27:79-92.

4. Malamut G, Cellier C. Refractory celiac disease. Expert Rev Gastroenterol Hepatol. 2014;8:323-328.

5. Kaukinen K, Lindfors K. Novel treatments for celiac disease: glutenases and beyond. Dig Dis. 2015;33:277-281.

6. Khaleghi S, Ju JM, Lamba A, Murray JA. The potential utility of tight junction regulation in celiac disease: focus on larazotide acetate. Therap Adv Gastroenterol. 2016;9:37-49.

7. Anderson RP, Jabri B. Vaccine against autoimmune disease: antigen-specific immunotherapy. Curr Opin Immunol. 2013;25: $410-417$.

8. Croese J, Giacomin P, Navarro S, et al. Experimental hookworm infection and gluten microchallenge promote tolerance in celiac disease. J Allergy Clin Immunol. 2015;135:508-516.

9. Sample DA, Sunwoo HH, Huynh HQ, et al. AGY, a Novel egg yolk-derived anti-gliadin antibody, is safe for patients with celiac disease. Dig Dis Sci. (Epub ahead of print). doi:10.1007/s10620016-4426-5.

10. Guandalini S, Polanco I. Nonceliac gluten sensitivity or wheat intolerance syndrome? J Pediatr. 2015;166:805-811. 